\title{
Torque Pull-Out Load Characteristics of Skull Traction Calipers of 'Ice Tong' Type (with particular reference to the Cone Type of Caliper) Technical Note
}

\author{
R. J. Minns, B.Eng., M.Sc., Ph.D. ${ }^{1}$ and R. A. Sutton, F.R.C.S., Ed ${ }^{2}$ \\ ${ }^{1}$ Senior Physicist, Regional Medical Physics Department, Durham Unit, Dryburn \\ Hospital, Durham DH1 5 TW. ${ }^{2}$ Consultant Orthopaedic Surgeon, North of England \\ Regional Spinal Injuries Unit, Hexham General Hospital, Hexham, Northumber- \\ land, U.K.
}

\begin{abstract}
Summary
The relationship between the applied torque and the load required to pull off the calipers was established on cadavers. Torques of 0.7, 0.8 and 0.9 Newton-metres were applied to a specially adapted skull traction caliper whilst attached to the skull in the normal position when used clinically, and loads were applied in steps of $2 \mathrm{lb}$ up to a maximum of $60 \mathrm{lb}$. It was found that at a torque of 0.7 Newton-metres, the caliper became detached at the maximum load, but still held during traction at torques above this value. Taking into account the fall in load with time that may occur clinically and the limiting torque for pull-out with a traction load of $60 \mathrm{lb}$, it is suggested for complete clinical safety that a torque of 0.9 Newton-metres should be applied. An open-ended torque release spanner was set for 0.9 Newton-metres for clinical use such that the compressive load is sufficient to prevent pull-out even in the unlikely event of $60 \mathrm{lb}$ being applied for up to 21 days.
\end{abstract}

Key words: Skull traction; Calipers; Compression; Evaluation and application.

\section{Introduction}

Skull traction calipers of various designs have been used for the application of a tensile loading on the spine to restore spinal alignment following trauma. The design of these calipers has varied in respect of the method and position of attachment to the skull. The Crutchfield calipers utilise two prongs inserted obliquely into the skull over a narrow span (Crutchfield, 1933, 1954 and 1966). In order to work effectively and safely a very large compressive load must be applied to the skull to prevent the Crutchfield calipers from pulling out as the pins are angled to the traction force direction. In practice this caliper becomes 'pulled-out' relatively frequently (Grundy, 1983).

The designs which utilise pins applied to the sides of the skull at right angles 
to the traction force direction are mechanically much more attractive. The 'Vinke' and 'Blackburn' type of designs are, in our opinion, unnecessarily complicated to apply (Vinke, 1948; Blackburn, 1938). The above mentioned calipers do not depend primarily on compressive force for stability under traction load.

The designs which utilise pins applied directly to the sides of the skull (i.e. without prior burr holes) enable application to be greatly simplified. The traction force in this situation is transferred to the skull surface by the fixing pin ends. 'Pull-out' of skull traction calipers of this type can occur and may be the result of either faulty application causing a poor mechanical fixation or local bone resorption around the pin ends resulting in a reduction of the compression and shear forces acting on the ends of the pins. No clinical studies have been published which describe the compression forces that exist during skull traction. Similarly, we are not aware of any published data which relates to the relationship between compressive force at the skull and pull-out due to increasing traction load.

The Cone type of caliper (Cone and Turner, 1937) is commonly used in Great Britain. We report on the traction force required to pull out the caliper of this design on cadavers. Of the various types of Cone calipers, we believe that the Stratford modification is the best, the pins are well supported in this model. Also, we suggest a simple torque spanner which is commercially available, set at a pre-determined torque to ensure reproducible and repeatable constant compression force applied to the skull through the calipers.

\section{Experimental Studies}

A single caliper of the 'Cone's' design was modified to not only incorporate a

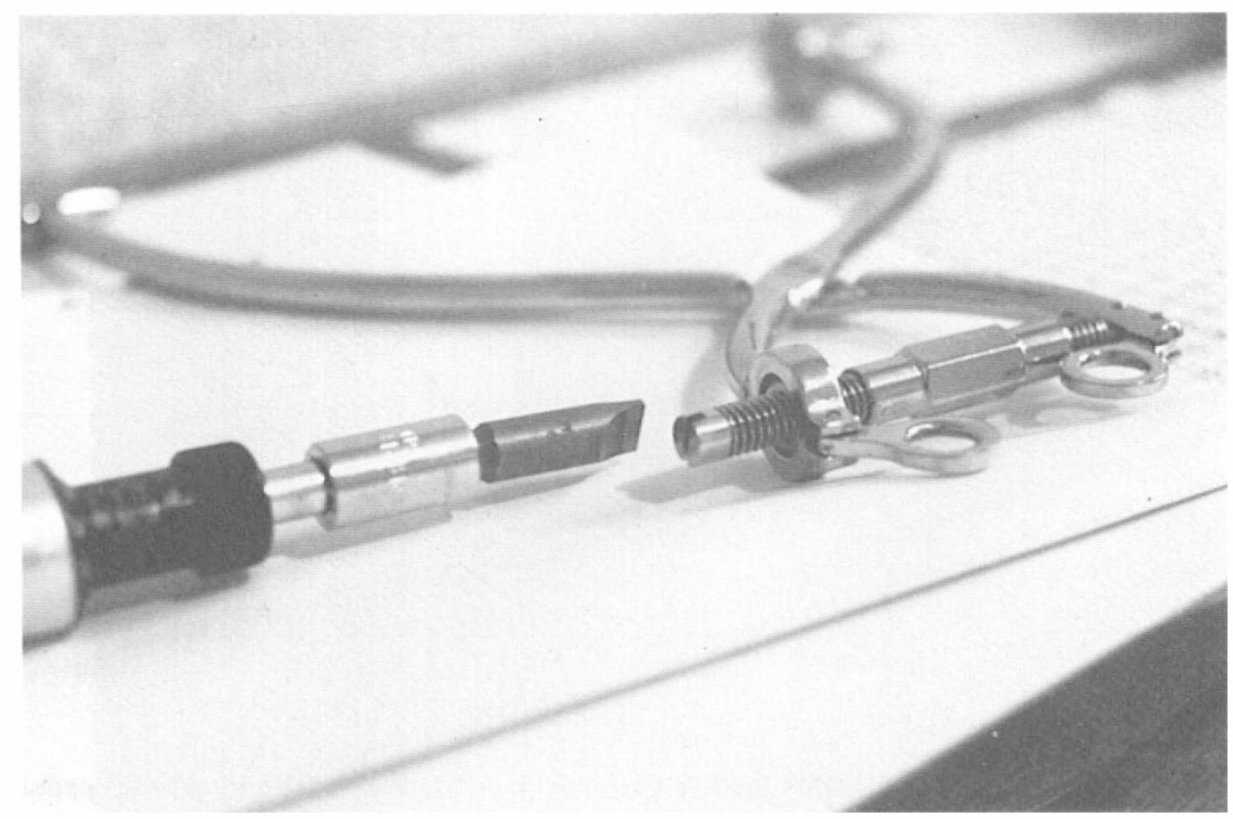

Figure 1. View of modified 'Cone' type calipers with adjustable torque screwdriver on the left applying varying values of torque to the tightening screw. 
load cell to measure the compressive force across the skull, but also the tightening screw was extended through a threaded bearing ending with a screwdriver slot. An adjustable torque screwdriver ${ }^{\star}$ was used on this slot (Fig. 1) to establish a relationship between the tightening screw torque and pin compressive force such that predetermined torques could be applied to the caliper and the traction pull-out forces established on cadavers. Torques of $0.7,0.8$ and 0.9 Newtonmetres (corresponding to a compression force of 58, 77 and 103 Newtons respectively) were applied to the calipers whilst attached to the skull of cadavers in the normal position when used clinically.

Traction forces were applied in steps of $2 \mathrm{lb}$ (approximately 9 Newtons) up to a maximum of $60 \mathrm{lb}$ (approximately 265 Newtons). If 'pull-out' occurred, the calipers were reapplied in a nearby position. It was found that at a compressive force of $13 \mathrm{lb}$ ( $58 \mathrm{Newtons),} \mathrm{the} \mathrm{caliper} \mathrm{became} \mathrm{detached} \mathrm{at} \mathrm{the} \mathrm{maximum} \mathrm{traction}$ force of $60 \mathrm{lb}$ but still held at the compression forces tested above $17 \mathrm{lb}$ ( 77 Newtons) as shown in Figure 2. Clinically, an instrumented load cell was incorporated into one of the fixing pins on a Cone's type skull traction caliper and it was found that the compression load fell by 15 per cent over a period of 21 days of traction (Minns and Sutton, Submitted for publication).

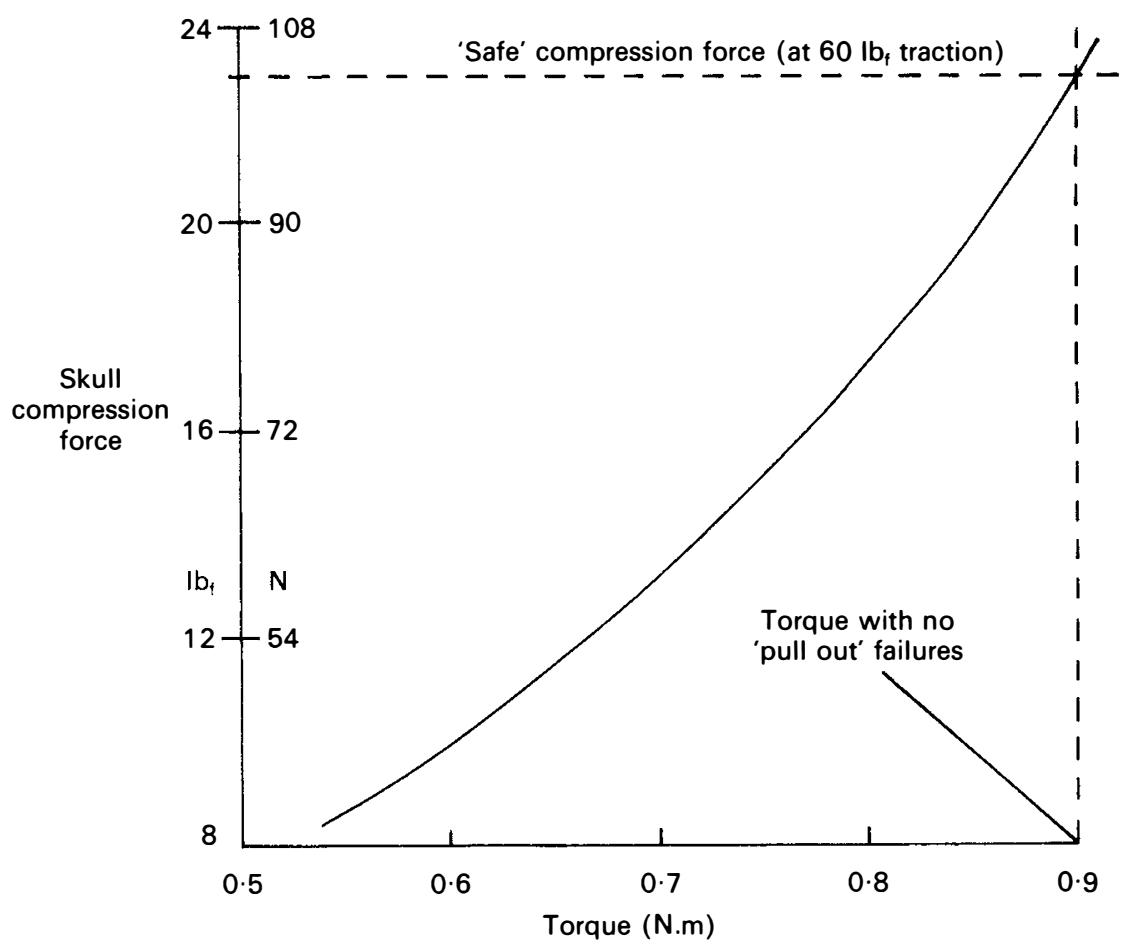

Figure 2. Variation of compressive force across the skull as detected by the load cell and applied torque.

^ Torque driver (minor). Code No. 609-354. R.S. Components Limited, Corby, Northants. 


\section{Conclusion}

In order to include a 'safety margin', a skull compression force of $23 \mathrm{lb}$ (103.5 Newtons) corresponding to a torque of 0.9 Newton-metres, was felt to be required when these calipers were used, which could be achieved by using a pre-set torque spanner ${ }^{\star}$ which broke' at a torque 0.9 Newton-metres (Fig. 3).
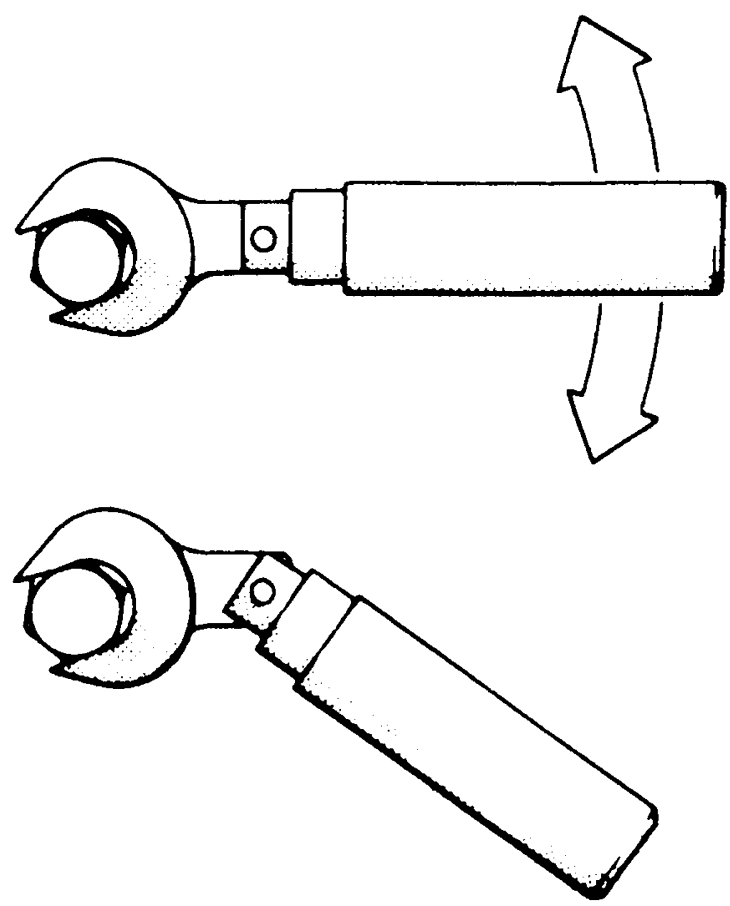

Figure 3. Torque release spanner which 'breaks' when a torque of $0.9 \mathrm{Nm}$ is reached producing a corresponding compressive force of $23 \mathrm{lb}$ across the skull.

On the basis of the above data, we have evaluated a commercially available torque-release spanner which will allow us to accurately apply a reproducible and repeatable force of $23 \mathrm{lb}$ (103.5 Newtons) to the skull. We believe that a spanner of this type would be of particular value to surgeons who apply skull calipers only occasionally, since the caliper would then be confidently expected to perform satisfactorily even up to traction loads of $60 \mathrm{lb}$. Calipers applied with this controlled degree of compression would also be able to resist the occasional transiently high 'impulse' traction forces encountered when patients are transferred from a district general hospital to a spinal injury unit. To obviate for the effect of bone resorption causing a lowering of compression, regular tightening with this spanner is also recommended, probably daily.

We believe that the use of a torque-release spanner in this way considerably enhances the value of the Cone type of ice tong calipers. 


\section{References}

Blackburn J 1938 A New Skull Traction Appliance, South Surgeon 7:16.

CONE W, TURNER WG 1937 The treatment of fracture-dislocations of the cervical vertebrae by skeletal traction and fusion. Journal of Bone and Joint Surgery 19:584-602.

CRutchfield WG 1933 Skeletal Traction for Dislocation of Cervical Spine, Report of a Case. South Surgeon 2:156-159.

CRUtchfield WG 1954 Skeletal Traction in Treatment of Injuries to the Cervical Spine. Journal of American Medical Association 155:29-32.

Crutchfield WG 1966 Redesigned Crutchfield Skull Tongs. Journal of Neurosurgery 25:656657.

GRUNDY DJ 1983 Skull Traction and its Complications. Injury 15:173-177.

VINKE TH 1948 A Skull-Traction Apparatus. Journal of Bone and Joint Surgery 30A:522-524. 